\section{Patent foramina ovale in elderly stroke patients}

Sir,

The finding by Vella and associates ${ }^{1}$ of the rarity of right-to-left shunting across a patent foramina ovale in elderly stroke patients is very important from the standpoint of patient management.

Contrast echocardiography before and after a Valsalva manoeuvre, which should be performed in every young adult stroke patient to exclude paradoxical embolism, ${ }^{2,3}$ need not be routinely performed in elderly stroke patients, because it is not cost-effective.

May I also suggest a more appropriate title for the article by Vella and associates be 'Paradoxical embolism in elderly stroke patients' than 'Patent foramina ovale in elderly stroke patients', because patent foramen ovale is just as common an anatomical finding in elderly people as in young people. ${ }^{1}$ Incidentally, the initials of my name, the author of their reference $8,{ }^{4}$ are T.O., not J.D.

Tsung $\mathrm{O}$. Cheng Professor of Medicine, The George Washington University Medical Center.

2150 Pennsylvania Avenue, N.W., Washington, DC 20037, USA.

\section{References}

1. Vella, M.A., Sulke, A.N., Rodrigues, C.A., McNabb, W.R. \& Lewis, R.R. Patent foramina ovale in elderly stroke patients. Postgrad Med J 1991, 67: 745-746.

2. Cheng, T.O. Echocardiography and paradoxical embolism. Ann Intern Med 1981, 95: 515.

3. Cheng, T.O. Echocardiography in ischaemic cerebrovascular disease. Br Med J 1987, 295: 856.

4. Cheng, T.O. Paradoxical embolism. A diagnostic challenge and its detection during life. Circulation 1976, 53: 565-568.

\section{Hypoglycaemia and atrial flutter}

\section{Sir,}

Recent case reports have noted an association between hypoglycaemia and atrial fibrillation in diabetic patients. ${ }^{1.2}$ We have also seen a patient who presented with hypoglycaemia caused by an insulinoma, with coincidental atrial flutter. A 70 year old male presented with a 6 month history of poor concentration which was provoked by fasting and relieved by eating. Investigation for a suspected insulinoma was initially equivocal and hypoglycaemia could not be provoked by fasting in hospital. He was re-admitted 2 years later with atrial flutter, and blood glucose was less than $2 \mathrm{mmol} / \mathrm{l}$ on testing with a reflectance meter. He was given oral dextrose and his blood glucose rose to normal within $30 \mathrm{~min}$. He reverted spontaneously to sinus rhythm $24 \mathrm{~h}$ after admission without any specific anti-arrhythmic therapy. Echocardiography and an exercise electrocardiogram (ECG) were normal. Angiography demon- strated a mass in the tail of the pancreas, and glucose:insulin ratios were raised confirming the clinical diagnosis of an insulinoma. The patient refused surgery, and during the following 2 years he had repeated episodes of hypoglycaemia and unconsciousness, with blood glucose $<2 \mathrm{mmol} / \mathrm{l}$ on testing with a reflectance meter. Paroxysms of atrial flutter/fibrillation were documented on several occasions. He finally agreed to surgery 4 years after presentation, and at operation the insulinoma was successfully removed. An ECG at the time showed sinus rhythm. One year after surgery he developed persistent atrial fibrillation, which has remained under control with digoxin. Post-operatively he has had no further episodes of hypoglycaemia after 5 years of follow-up, but he continues to have severe memory impairment.

In previous reports of insulin-induced hypoglycaemia and atrial fibrillation, sinus rhythm returned spontaneously within $1 \mathrm{~h}$ of the administration of dextrose,${ }^{1-3}$ but one patient was described who reverted to sinus rhythm after $24 \mathrm{~h} .{ }^{4}$ Whilst it is possible that our patient simply had the coincidental occurrence of the two diseases, on at least one occasion a temporal association was established between the occurrence of hypoglycaemia and atrial flutter, and he returned to sinus rhythm within $24 \mathrm{~h}$ of the correction of hypoglycaemia, without any specific antiarrhythmic therapy.

There are several possible mechanisms for hypoglycaemia-induced arrhythmias. Acute hypoglycaemia stimulates activation of the sympathetic nervous system which may cause increased ectopic activity. Acute hypoglycaemia is also associated with a fall in serum potassium which might provoke cardiac arrhythmias. However, these acute changes are not generally seen with an insulinoma, where the insidious onset of the metabolic abnormalities does not always provoke a distinct counterregulatory response. The heart utilizes glucose as a substrate and it is possible that the arrhythmia might have been provoked by the metabolic disturbance of tissue glucopenia affecting the myocardium. Because of the long duration of symptoms in this patient, with intermittent severe hypoglycaemia over several years, secondary irreversible structural damage may have developed, leading to eventual persistence of the arrhythmia.

B.M. Fisher, D.A.S. Marshall, A.C. MacCuish Diabetes Centre, Royal Infirmary, Glasgow G4 OSF, UK.

\section{References}

1. Baxter, M.A., Garewal, C., Jordan, R., Wright, A.D. \& Natrass, M. Hypoglycaemia and atrial fibrillation. Postgrad Med J 1990, 66: 981 (letter).

2. Collier, A., Mathews, D.M., Young, R.J. \& Clarke, B.F. Transient atrial fibrillation precipitated by hypoglycaemia: two case reports. Postgrad Med J 1987, 63: 895-897.

3. Goldman, D. The electrocardiogram in insulin shock. Arch Intern Med 1940, 66: 93-108.

4. Soskin, S., Katz, L.N., Strouse, S. et al. Treatment of elderly diabetic patients with cardiovascular disease. Arch Intern Med 1932, 51: $122-142$. 